\title{
FACTORES OBSTÉTRICOS ASOCIADOS A MORBILIDAD MATERNA EXTREMA EN EL HOSPITAL SANTA ROSA, ENERO - DICIEMBRE 2015
}

\author{
OBSTETRIC RISK FACTORS ASSOCIATED WITH EXTREME MATERNAL \\ MORBIDITY AT SANTA ROSA HOSPITAL, JANUARY - DECEMBER 2015 \\ Diana Celeste Baltodano-Garcíala, , Jorge Burgos-Miranda a,a,b
}

\begin{abstract}
RESUMEN
Objetivos: Determinar la asociación entre factores obstétricos y el desarrollo de morbilidad materna extrema en gestantes atendidas en el Hospital Santa Rosa durante el 2015. Métodos: El tipo de estudio es observacional, analítico, longitudinal, de casos y controles y retrospectivo. La población es de 2016 gestantes. La muestra es de 102 gestantes que cumplían criterios de inclusión y exclusión, por lo que no fue aleatorio. La información fue obtenida a partir de historias clínicas, y se seleccionaron 51 casos y 51 controles a partir de una ficha de datos. Las variables fueron evaluadas mediante un análisis bivariado y multivariado. Resultados: Los factores asociados fueron nuliparidad OR ajustado 8,12 (IC 95\% 2.40-27.43), cesárea previa OR ajustado 4,55 (IC95\% 1,07-19,3), periodo intergenésico corto OR ajustado 7,60 (IC 95\% 2,12- 27,23) y controles prenatales inadecuados OR ajustado 3,23 (IC95\% 1,03-10,04). Conclusión: Los factores nuliparidad, cesárea previa, periodo intergenésico corto y control prenatal inadecuado se asociaron al desarrollo de esta patología en gestantes atendidas en el Hospital Santa Rosa durante el año 2015.
\end{abstract}

Palabras clave: Morbilidad materna extrema; Nuliparidad; Cesárea previa; Periodo intergenesico corto; Control prenatal. (fuente: DeCS BIREME)

\begin{abstract}
Objective: To determine the association between obstetric factors and maternal near miss in pregnant women atended at Santa Rosa Hospital during 2015. Methods: The type of study is observational, analytical, longitudinal, case-control and retrospective. The population is 2016 pregnant women. The sample was 102 pregnant women that satisfied inclusion and exclusion criteria, so it was not randomized. Data was obtained from clinical histories and were selected 51 cases and 51 controls, information was collected in a data sheet. Bivariate and multivariate analyses of variables were performed. Results: The variables associated were nulliparity OR 8,12 (IC 95\% 2.4027.43), previous cesarean section OR 4,55 (IC 95\% 1.07-19.3), short intergenesic period OR 7,60 (IC95\% 2,1227,23), and a inadequate prenatal care OR 3,23 (IC 95\% 1,03-10,04). Conclusions: The factors: nulliparity, previous cesarean section, short intergenesic period,and inadequate prenatal care were associated with the development of maternal near miss in pregnant women atended at Santa Rosa Hospital during 2015.
\end{abstract}

Key words: Maternal near miss; Nulliparity; Previous cesarean section; Short intergenesic period; Prenatal care. (source: MeSHNLM)

\footnotetext{
'Internado Médico del Hospital Santa Rosa, Lima Perú.

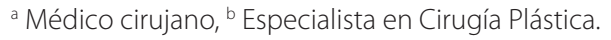

Correspondencia: Diana Celeste Baltodano García. Celular: 942652717. Correo: qwe_273@hotmail.com
}

Citar como: Diana Celeste Baltodano-García, Jorge Burgos-Miranda. Factores obstétricos asociados a morbilidad materna extrema en el Hospital Santa Rosa, enero-diciembre 2015. [Artículo Original]. Rev. Fac. Med. Hum. 2017;17(1):43-47. DOI 10.25176/RFMH.v17.n1.747 


\section{INTRODUCCIÓN}

La mortalidad materna es uno de los temas de mayor problemática en el sector de la salud. Es por ello que figura dentro de las prioridades nacionales la reducción en el número de casos para el año 2020; y ha sido considerado uno de los objetivos del milenio de la Organización Mundial de la Salud OMS hacia el año 2015.

Los índices de mortalidad materna y de morbilidad materna extrema son importantes de ser evaluados, ya que son un reflejo de la calidad de atención sanitaria.

A partir de 1991 en Reino Unido, Stones es el primero en usar el término para referirse a aquellas gestantes que tuvieron una condición clínica que amenazaba su vida, pero que sobrevivieron; y su importancia radicaba en las poblaciones donde el índice de mortalidad era bajo y no podían definirse por completo los factores involucrados en el evento.

La reducción en los factores de riesgo involucrados en morbilidad materna extrema, es por tanto de importancia en el bienestar materno fetal.

\section{MÉTODOS}

Se realizó un estudio de tipo observacional, analítico, longitudinal, de casos y controles y retrospectivo en el servicio de Ginecología y Obstetricia del Hospital Santa Rosa localizado en Lima, Perú; durante el periodo 2015 con el cumplimiento de los siguientes criterios de inclusión y exclusión.

\section{Criterios de inclusión para los casos:}

- Pacientes con compromiso vital por toxemia durante su embarazo o puerperio inmediato.

- Pacientes con compromiso vital por hemorragia obstétrica severa durante su embarazo o puerperio inmediato.

- Pacientes con compromiso vital por sepsis severa durante su embarazo o puerperio inmediato.

- Mujeres con compromiso vital por disfunción orgánica durante su embarazo o puerperio inmediato.

- Pacientes con compromiso vital que requirieron ingreso a UCl durante su embarazo o puerperio inmediato.

- Pacientes con compromiso vital que requirieron procedimiento quirúrgico de emergencia durante su embarazo o puerperio inmediato.

- Pacientes con compromiso vital que requirieron transfusión sanguínea durante su embarazo o puerperio inmediato.

\section{Para los controles:}

- Gestante o puérpera inmediata que no presentó compromiso vital.

\section{Criterios de exclusión}

- Gestantes que reciban el alta antes del término de la gestación.

- Gestantes que no cumplan con el diagnóstico de morbilidad materna extrema.

- Gestantes que no cumplan con un registro completo de historia clínica.

- Gestantes que ingresen con diagnóstico de comorbilidad pre gestacional.

- Gestantes que fallezcan durante su estancia hospitalaria.

Se elaboró una ficha de recolección de datos que contenía las variables para evaluar los objetivos planteados. La fuente de información fue indirecta, ya que se recabo a partir de las historias clínicas.

\section{RESULTADOS}

La muestra estudiada de 102 pacientes presentaba una edad promedio de 26,1 años \pm 8.27 , con una mediana de 2,45 y una moda de 19,0 años. En el grupo de casos se encontró 18-35 años (60.8\%), menores de 18 años (11.8\%), mayor a 35 años (27.5\%). La distribución fue similar en los controles, $68.97 \%$ 18-35 años, $14.66 \%$ menores de 18 años, y 16,38\% mayores a 35 años.

La causa predominante se atribuye a preeclampsia severa, $(62.75 \%), 15.69 \%$ a hemorragia severa, $9.8 \%$ a eclampsia, 3.92\% a embarazo ectópico, 3.92\% a shock séptico (foco renal), $1.96 \%$ a shock séptico (foco neumónico), $1.96 \%$ a shock séptico puerperal.

Por enfermedad específica, la causa predominante fue shock hipovolémico, (47.06\%), eclampsia (29.41\%), shock séptico (23.53\%).

En relación a la condición de nuliparidad se obtuvieron los siguientes resultados.

\section{DISCUSIÓN}

El presente estudio de casos y controles fue desarrollado en el Hospital Santa Rosa, donde se registró a lo largo de un año de investigación 51 casos que cumplían los criterios de inclusión, con una media de edad de 26,1 años \pm 8.27 , predominando el grupo entre los $18-35$ años, la causa se atribuye a trastornos hipertensivos en un $66.67 \%$, siendo la más frecuente la preeclampsia severa, $62.75 \%$, en segundo lugar se ubicó las hemorragias 


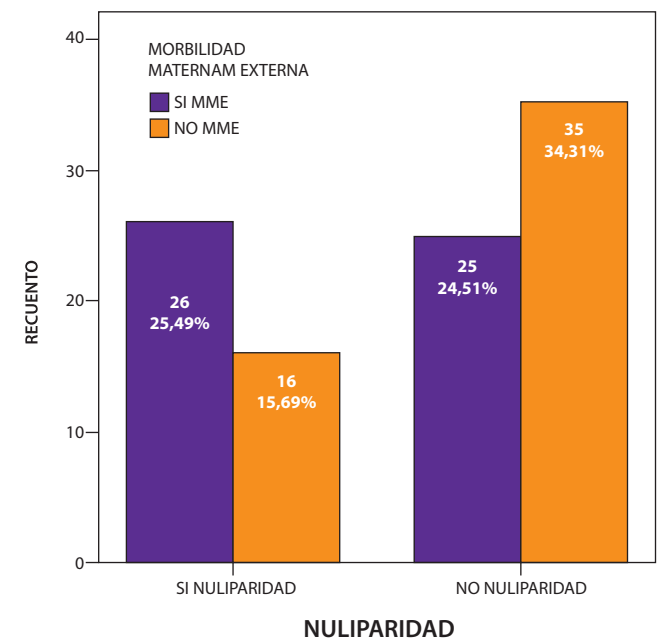

Fuente: INICIB-FAMURP

Gráfico 1. Factores obstétricos asociados a morbilidad materna extrema.

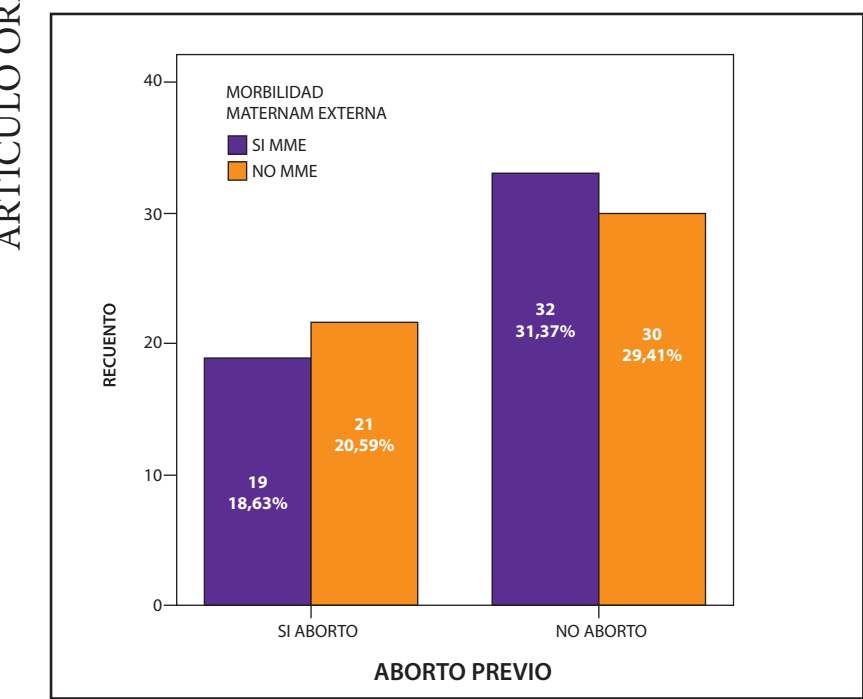

Fuente: INICIB-FAMURP

Gráfico 2. Factores obstétricos asociados a morbilidad materna extrema.

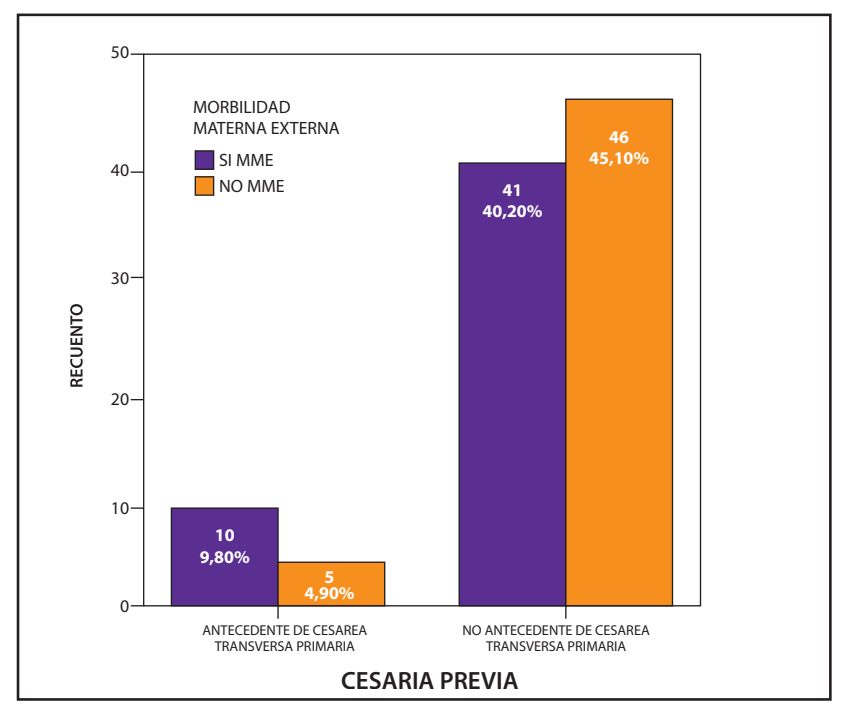

Fuente: INICIB-FAMURP

Gráfico 3. Factores obstétricos asociados a morbilidad materna extrema.

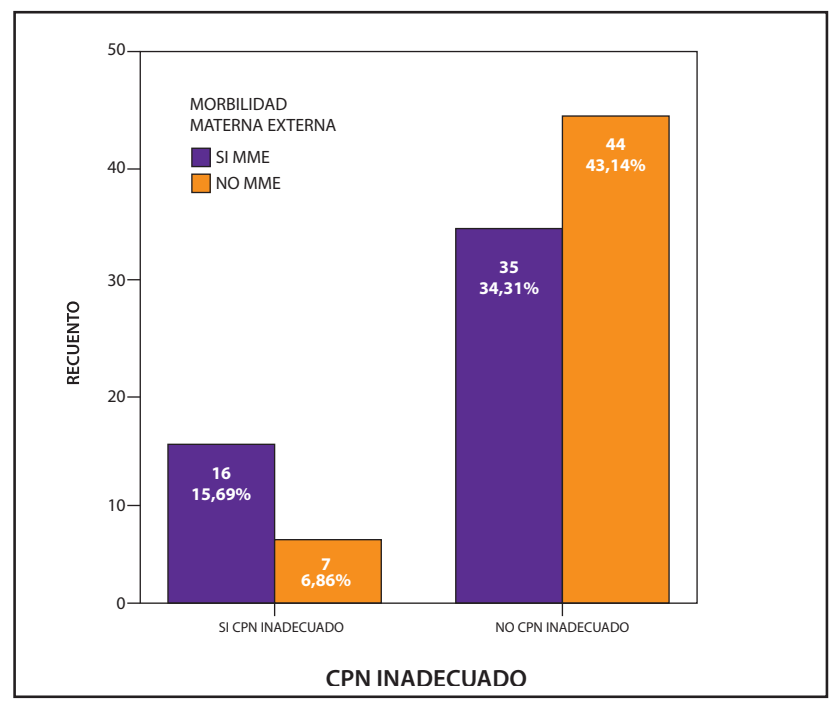

Fuente: INICIB-FAMURP

Gráfico 4. Factores obstétricos asociados a morbilidad materna extrema.

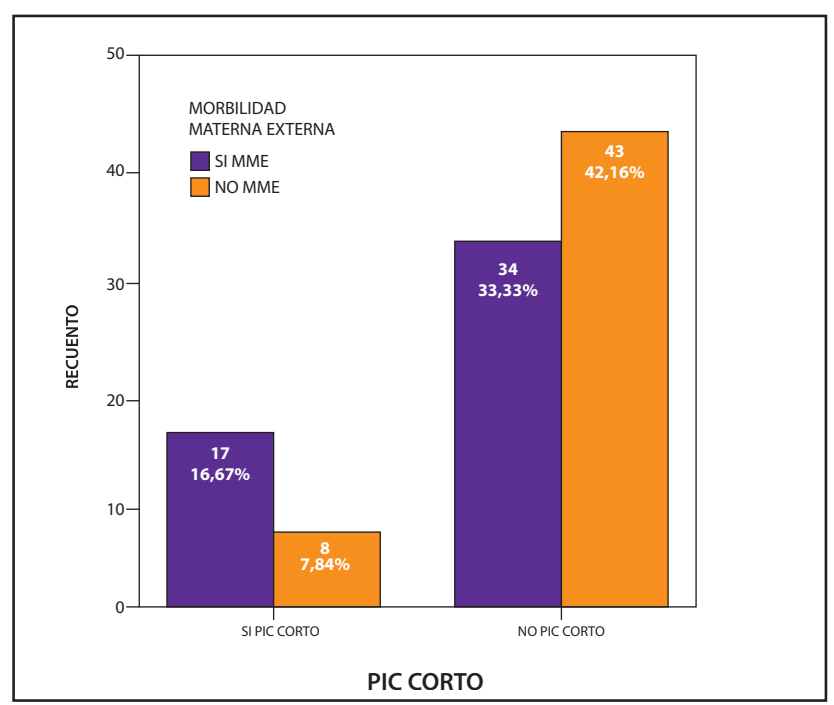

Fuente: INICIB-FAMURP

Gráfico 5. Factores obstétricos asociados a morbilidad materna extrema.

postparto por atonía uterina en un $15.69 \%$, un 3.92\% fueron causadas por embarazo ectópico, y un $7.84 \%$ por shock séptico de causas obstétricas y no obstétricas, hallándose más casos no obstétricos.

Resultado similar a la investigación desarrollada por Bendezú et al en el año 2012 en un Hospital de Ica, Perú; durante 2006-2012 se identificó 58 casos con una media de edad de 30,67 años \pm 6.03 , y 67.2\% de multigrávidas frente a las nulíparas (51\%) ${ }^{1}$.

En un estudio desarrollado por Costa durante el año 2015 en Brasil; los trastornos hipertensivos (62.7\%) fueron la causa predominante desarrollándose trombocitopenia en su mayoría, al igual que el resultado obtenido en este estudio. También coincidieron las edades entre 20-34 años que representaron un $66.7 \%$ de casos $^{2}$.

En un estudio realizado por Carrillo et al., en 2014 
en Colombia, se encontró 353 casos y como causas generales los trastornos hipertensivos en un 55.24\%, complicaciones hemorrágicas en un $21.25 \%$; la edad promedio también entre los 18-35 años (58.93\%); el
44.19\% eran nulíparas, y 55.81\% tenían un periodo intergenésico menor a un año, $32.86 \%$ no tenía controles prenatales previos ${ }^{3}$.

Tabla 1. Factores obstétricos asociados a morbilidad materna extrema.

\begin{tabular}{llll}
\hline Variable & OR & IC 95\% & Valor P \\
\hline Nulipara & 8.129 & $2.40-27.43$ & $\mathrm{p}=0.01$ \\
\hline Aborto Previo & 0.27 & $0.27-1.77$ & $\mathrm{p}=0.45$ \\
\hline Cesarea Previa & 4.55 & $1.07-19.3$ & $\mathrm{p}=0.04$ \\
\hline Controles Prenatales $<6$ & 3.23 & $1.03-10.04$ & $\mathrm{p}=0.04$ \\
\hline Periodo Intergenesico Corto & 7.60 & $2.12-27.23$ & $\mathrm{p}=0.00$ \\
\hline Gestante Añosa & 2.26 & $0.72-7.05$ & $\mathrm{p}=0.15$
\end{tabular}

Fuente: INICIB-FAMURP

La nuliparidad fue el factor obstétrico más asociado. (OR ajustado =8.129 IC 2.40-27.43).

Resultado compatible con un estudio realizado por Gray et al en el año 2012 en EEUU; donde nuliparidad y multiparidad se hallan asociadas; nuliparidad con un OR 1,83 (IC $95 \%$ 1,72-1,95)

La cesárea previa representa un 19,6\% dentro del grupo de casos y se asocia al evento. (OR ajustado $=4,55$ IC 1,07 - 19,3).

Resultado compatible con el estudio realizado por González et al en el año 2015, desarrollado en una unidad de cuidados intensivos, Cuba. La población fue de 504 pacientes, y se evalúa una muestra de 153 pacientes, el antecedente de cesárea se asocia con un OR ajustado de 2,02 (IC $95 \%$ 1,1-3,6) ${ }^{5}$.

En un estudio realizado por Gray et al en el año 2012, en el estado de Washington, EEUU, se contó con una muestra de 9485 pacientes, donde se halló un OR de nuliparidad de 2.08 (IC 95\% 1.93 - 2.23) .

En un estudio multicéntrico realizado por Paulo et al en el año 2010, en 120 hospitales de América Latina durante tres meses, Brasil en una población de estudio de 97095 gestantes y una muestra de 2964, 4\% de ellas presenta cesárea previa y OR ajustado de 1.63 (IC $95 \% 1.47-1.81)^{6}$.

El periodo intergenésico corto representa un 33,3\% dentro del grupo de casos y se asocia al evento. (OR ajustado $=7.60 \mathrm{IC}$ 2.12-27.23).

Resultado compatible con un estudio realizado por Habimana et al en el año 2015, que asocia el periodo intergenésico a morbilidad materna extrema en Rwanda. La población consta de 6325 pacientes, un periodo intergenésico corto (considerado en este estudio menor a doce meses) representa un 22,1\% de los casos, se asocia con un OR 1,67 9 .

El antecedente de número de controles prenatales menores a seis $(\mathrm{CPN}<6)$ representa un $31,4 \%$ de los casos y se asocia al evento. (OR ajustado $=3.23$ IC 1.03-10.04).

Resultado compatible con un estudio realizado por Correia et al en 2014, en el Hospital Dom Malan, Brasil. La población consta de 2291 pacientes, y se tiene una muestra de 400 pacientes, un número de controles prenatales menores a seis se asocia a un OR ajustado de 1,13 (IC $95 \%$ 1,01-1,69) 7 .

Se evidencia un resultado similar, en un estudio realizado por Aparecida et al, en el año 2012, en una unidad de cuidados intensivos, Brasil; con una población de 158 y muestra de 43 casos, donde un $28,2 \%$ presenta la condición de $\mathrm{CPN}<6$ con un OR ajustado de 4.44 (IC 95\% 0.67-29.53) ${ }^{8}$.

El aborto previo representa un $37,3 \%$ de los casos, y no se asocia al evento. (OR ajustado $=0.27$ IC 0.27-1.77).

El resultado contrasta con estudios revisados como el realizado por Assarag et al en el 2015, en, Indiana, la población era de 299 pacientes y la muestra de 80 casos, un $21 \%$ de ellos se asociaban a abortos $\geq 1$, teniendo un OR 3.67 (1.73-7.77) 45.

La condición de gestante añosa representa un 26,7\% dentro del grupo de casos, y es independiente al evento. (OR ajustado =1,35 IC 0,68 - 2,67), en la Revista 
Peruana de Epidemiología, el grupo de casos con edad > 35 años solo representa un 10,7\%, en el análisis bivariado no se establece asociación y se concluye como factor de riesgo la primigravidad (OR:1.54; IC95\%:1.3-8.72; <0.01) condición de las gestantes <35 años (89.3\% de casos de la muestra).

Sin embargo, el estudio realizado por Valdés en el 2013, en Cuba. La población fue de 128 pacientes, 64 casos y 64 controles, las edades de 15-19 años obtuvieron un OR de 3,11 (IC 95\% 0,85 - 14,10\%) y el tener más de 35 años se asoció a un OR de 4,27 (IC 95\% 1,37 - 15,74\%). Por otro lado, en el estudio realizado por Paulo et al en el año 2010, Brasil un 4\% presenta una edad superior a 35 años (>35) y se asocia con un OR ajustado de 1.40 (IC 95\% 1.20-1.64). Las características y distribución de ellas en la población y muestra son muy alejadas de la nuestra, además que toma en cuenta ocho países con características sociodemográficas y antecedentes obstétricos no equitativos ${ }^{6}$.

En investigaciones fuera de América Latina, también difiere, en un estudio realizado por Gray et al en el 2012, EEUU, contando con una población de 50597 y una muestra de 9485 pacientes, aquellas con edades en intervalo de 35-39 se asocian al evento con un OR de 1.65 (IC 95\% $1.52-1.79)^{4}$.

Financiamiento: Autofinanciado.

Conflicto de interés: Los autores declaran no tener conflictos de interés en la publicación de este artículo.

Recibido: 01 de marzo del 2017

Aprobado: 16 de marzo del 2017

\section{REFERENCIAS BIBLIOGRÁFICAS}

1. Bendezú G. Caracterización de la morbilidad materna extremadamente grave en un Hospital del Seguro Social del Perú. Revista Peruana de Ginecología y Obstetricia. 2014

2. Costa L, Ribeiro A. Maternal near miss in the intensive care unit: clinical and epidemiological aspects. Rev. Bras. Ter Intensiva. 2015; 27(3):220-227.

3. Carrillo J, García C. Comportamiento de la morbilidad materna extrema en el departamento del Meta, Colombia, 2014. Hacia promoc. Salud. 2016; 21(1):15-25

4. Gray E. K., Wallace E.R., Nelson R. K., Reed D.S., Schiff A. M.: PopulationBased Study of Risk Factors for Severe Maternal Morbidity. EEUU. Paediatric and Perinatal Epidemiology 2012, Vol.26(6) 506-514.

5. González Aguilera J., Vázquez Belizón Y., Pupo Jiménez J., Algas Hechevarría L., Cabrera Lavernia J.: Extreme maternal morbidity in an intensive care unit. Cuba. MEDISAN 2015, 19(12):1466- 1476.
6. Paulo Souza J., Guilherme Cecatti J., Faundes A., Siani Morais S., Villar J., Carroli G., Gulmezoglu M., Wojdyla D., Zavaleta N., Donner A., Velazco A., Bataglia V., Valladares E., Kublickas M., Acosta A: Maternal near miss and maternal death in the World Health Organization's 2005 global survey on maternal and perinatal health. Bull World Health Organ 2010. Vol. 88:113-119.

7. Correia Pacheco J., Katz L., Rolland Souza A., Ramos de Amorim M.: Factors associated with severe maternal morbidity and near miss in the Sao Francisco Valley, Brazil : a retrospective, cohort study. Brazil. BMC Pregnancy and chillbirth 2014, 14:91.

8. Aparecida Lotufo F., Parpinelli M., Maerrawi Haddad S., Garanhani Surita F., Guilherme Cecatti J.: Applying the new concept of maternal near-miss in an intensive care unit. Brazil. CLINICS 201; Vol.67(3):225-230.

9. Assarag B., Dujardin B., Delamou A., Meski Z., De Brouwere V.: Determinants of Maternal Near-Miss in Morocco: Too Late, Too Far, Too Sloopy. Morocco. PLOS ONE 2015.

Consulte la Versión Electrónica de la Revista: Facultad de Medicina Humana Universidad Ricardo Palma http://revistas.urp.edu.pe/index.php/RFMH<smiles>C1=CCCC1</smiles>

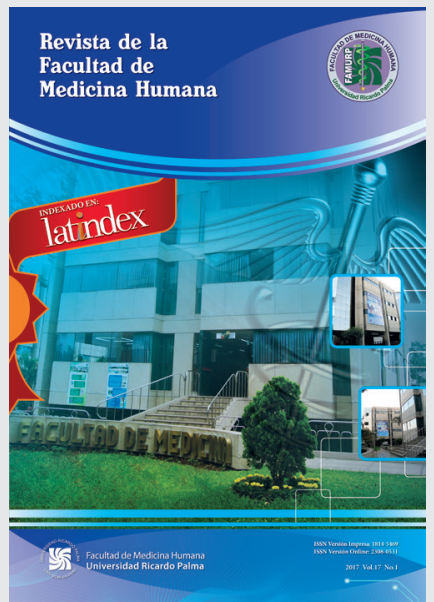

